University of Nebraska - Lincoln

DigitalCommons@University of Nebraska - Lincoln

11-1-1995

\title{
High resolution electron microscopy and nano-probe study of CoSm/Cr films
}

Yi Liu

University of Nebraska-Lincoln, yliu@unl.edu

David J. Sellmyer

University of Nebraska-Lincoln, dsellmyer@unl.edu

B. W. Robertson

University of Nebraska-Lincoln, brobertson1@unl.edu

Z.S. Shan

University of Nebraska - Lincoln

Sy_Hwang Liou

University of Nebraska-Lincoln, sliou@unl.edu

Follow this and additional works at: https://digitalcommons.unl.edu/physicsliou

Part of the Physics Commons

Liu, Yi; Sellmyer, David J.; Robertson, B. W.; Shan, Z.S.; and Liou, Sy_Hwang, "High resolution electron microscopy and nano-probe study of CoSm/Cr films" (1995). Si-Hwang Liou Publications. 59.

https://digitalcommons.unl.edu/physicsliou/59

This Article is brought to you for free and open access by the Research Papers in Physics and Astronomy at DigitalCommons@University of Nebraska - Lincoln. It has been accepted for inclusion in Si-Hwang Liou Publications by an authorized administrator of DigitalCommons@University of Nebraska - Lincoln. 


\title{
HIGH RESOLUTION ELECTRON MICROSCOPY AND NANO-PROBE STUDY OF CoSm // Cr FILMS
}

\author{
Y. Liu ${ }^{1,2}$, D. J. Sellmyer ${ }^{1,3}$, B. W. Robertson ${ }^{1,2}$, Z. S. Shan ${ }^{1,3}$ and S. H. Liou ${ }^{1,3}$ \\ ${ }^{1}$ Center for Materials Research and Analysis, ${ }^{2}$ Department of Mechanical Engineering, \\ 3 Behlen Laboratory of Physics, University of Nebraska, Lincoln, NE 68588.
}

Abstract: The crystal structure of the crystallites in $\mathrm{CoSm}$ thin films deposited on $\mathrm{Cr}$ underlayer was studied by nanodiffaction and high resolution electron microscopy (HREM). It was found that the crystallites have a closed-packed structure. Some nanodiffaction patterns taken from different crystallites using a two nanometer probe can be indexed by two layer stacking $A B$ (HCP structure), three layer stacking $\mathrm{ABC}$ (FCC structure), and four layer stacking ABAC (double hexagonal structure), suggesting that a particular local stacking mode could exist. [1120] HREM images confirmed that the stacking sequence changes within one crystallite. In local regions, random stacking, and unit cells of two layer stacking $A B$, three layer stacking $\mathrm{ABC}$, and four layer stacking $\mathrm{ABAC}$ were found.

\section{INTRODUCTION}

The next generation of longitudinal recording media requires a recording density of $10 \mathrm{~Gb} / \mathrm{in}^{2}$ which means that the bit size is on the order of a few hundred nanometers. In order to meet such requirements; the recording media should have high coercivity, large anisotropy and small grain size (10 nm or smaller). Magnetic thin films sputtered from the CoSm system have been shown to be promising as recording media. High coercivities of 2-3 kOe [1] and recently up to $4.0 \mathrm{kOe}$ [2] have been obtained from $\operatorname{CoSm}$ films.

Recent microstructural characterization of CoSm films deposited by DC magnetron sputtering has shown that the microstructure of a CoSm thin film is composed of an amorphous matrix and crystallites with a grain size of about $5 \mathrm{~nm}$ [3]. Some CoSm thin films of small thickness ( $24 \mathrm{~nm}$ or smaller) deposited on the $\mathrm{Cr}$ underlayer at argon pressures of $12 \mathrm{mTorr}$ (or higher) display grain-like contrast of about $25 \mathrm{~nm}$. Such contrast was found to be inherited from the $\mathrm{Cr}$ underlayer [4]. As different sputtering processes have different dynamics for the formation of the nanostructure, it is important to identify the structure of the crystallites. In this paper we describe a detailed study of the structure of the crystallites in CoSm films by nanodiffraction and high resolution electron microscopy (HREM). Such knowledge of the crystal structure will provide useful information for understanding the magnetic properties of the films.

\section{EXPERIMENTAL PROCEDURE}

The $\mathrm{CoSm}$ thin films were prepared by $\mathrm{DC}$ magnetron sputtering at pressures of 5- 30 mTorr. First, about $95 \mathrm{~nm}$ of $\mathrm{Cr}$ was deposited on a $0.22 \mathrm{~mm}$ thick glass substrate. The CoSm films of $24 \mathrm{~nm}$ were then deposited on the $\mathrm{Cr}$ layer under the same argon pressure. The average composition of the films found using energy dispersive X-ray spectroscopy (EDX) in a scanning electron microscope was $79 \mathrm{at} \% \mathrm{Co}, 21 \mathrm{at} \% \mathrm{Sm}$, which is very close to the target composition of $\mathrm{Co}_{4} \mathrm{Sm}$. Samples of three millimeters in diameter were drilled from the specimens for planview transmission electron microscopy (TEM) examination. The disks were then dimpled from the substrate side to a thickness of $5 \mu \mathrm{m}$ at the center. Ion milling followed until perforation was just achieved. The TEM was performed using a JEOL 2010 transmission electron microscope operating at $200 \mathrm{kV}$.

\section{RESULTS}

Figure 1 is a plan-view HREM image of the CoSm film processed at 5 mTorr of argon gas. The size of each crystallite is identified by the lattice fringes of the same style. The volume fraction of the crystallites against amorphous phase is about $91 \%$ as measured by the nanodiffraction technique [3]. The crystallite that is aligned on the [1120] zone axis is marked $\mathrm{c}$ for higher magnification ( see Figure 6). The powder electron diffraction patterns taken from the CoSm films cannot be matched to any existing phases in the CoSm binary system.

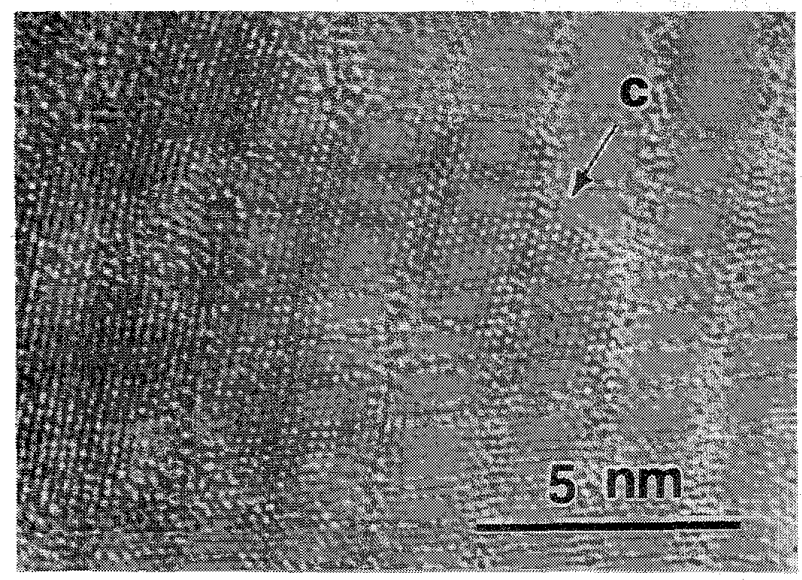

Figure 1. HREM micrograph showing the crystallites in the CoSm film.

The crystal structure of the crystallites was studied by nanodiffraction. The electron probe size is estimated to be about $2 \mathrm{~nm}$. About twenty nanodiffraction patterns were analyzed. It was found that these patterns can be indexed by models based on a close-packed structure with different stacking sequences. The zone axes for indexing are shown in Figure 2. The patterns are 
divided into two groups and are shown in Figure 3 and Figure 4. The group shown in Figure 3 can be indexed by zone axes from

(a) through (d) shown in Figure 2. These patterns all have (1210) spot in common. The nanodiffraction pattern simulations were performed using a computer package EMS [5]. As the thickness of the crystallite is small $(5 \mathrm{~nm})$, the diffracted beams are very weak compared to the transmitted beam. The central spot was attenuated by a factor of $10-100$ in the simulated patterns depending on the zone axis. The intensity of the diffraction spots in some zone axes such as [1010] are not sensitive to the stacking mode. Quite similar [10ī0] zone axis patterns can be obtained from all the stacking modes. Therefore we acknowledge that the matching of the TEM patterns and simulated patterns for

some zone axes such as [10ī0] does not suggest a unique identification of a particular stacking mode but proves that the TEM patterns can be matched to a model based on the closepacked structure.

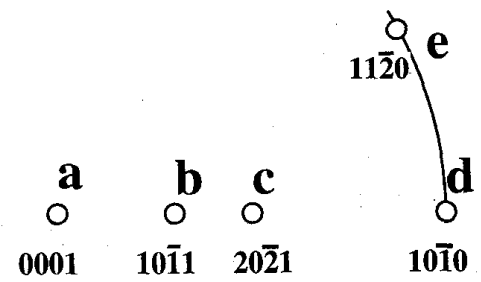

Figure 2. Stereo projection showing the zone axes for nanodiffraction pattern matching.

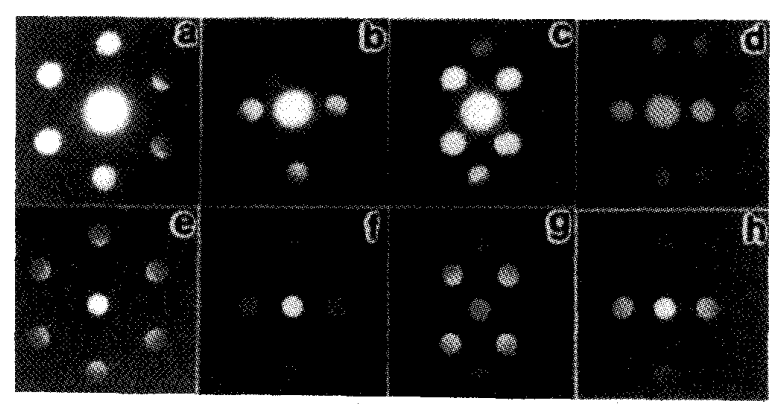

Figure 3. Comparison of TEM ( $a, b, c$, and d) and simulated (e,f, $\mathrm{g}$ and $\mathrm{h}$ ) nanodiffraction patterns. (a) and (e) : [0001] zone axis, (b) and (f) : [10ī1] zone axis, (c) and $(\mathrm{g}):$ [202̄1] zone axis, (d) and (h) : [1010] zone axis. The simulations were performed using a three layer model $A B C$ stacking for (e), and four layer stacking $A B A C$ for $(\mathrm{f})$, (g) and (h).

The second group of nanodiffraction patterns can be indexed by the $[11 \overline{2} 0]$ zone axis (Figure 4$)$. The [1120] zone axis nanodiffraction patterns lead to a decisive identification of the stacking mode. The stacking period can be deduced in the diffraction pattern in the following way [6]. Supposing the stacking period is $\mathrm{N}$ layers, reflections $(000 \ell)$ where $\mathscr{L} \neq \mathrm{i} \bullet \mathrm{N}$ (i is an integer) have zero structure factors while reflections $(000 \ell)$ where $l=\mathrm{i} \bullet \mathrm{N}$ have large structure factors. The stacking sequence is identified by the intensity distribution in the diffraction pattern. In this way, the stacking modes $A B$ (Figure 4 (b)), ABC (Figure 4 (c)), and ABAC (Figure 4 (d)) are identified. In many cases, a pattern with overlapping spots was obtained, suggesting a random stacking as shown in Figure 4 (a). The simulated pattern corresponding to Figure 4 (a) was based on a 9layer stacking mode to show that the overlapped spots indeed can be generated by a random stacking ( a random stacking can be considered to be $\mathrm{N}$-layer stacking as $\mathrm{N}$ tends to infinity). Lattice parameters $a=0.245-0.256 \mathrm{~nm}, \mathrm{c} 0=0.204-0.207 \mathrm{~nm}$ ( $\mathrm{c} 0$ is the spacing of the close-packed planes, $\mathrm{c}=\mathrm{N} \cdot \mathrm{c} 0$ for a $\mathrm{N}$ layer stacking unit cell ) were deduced from nano-diffraction patterns shown in Figure 4.

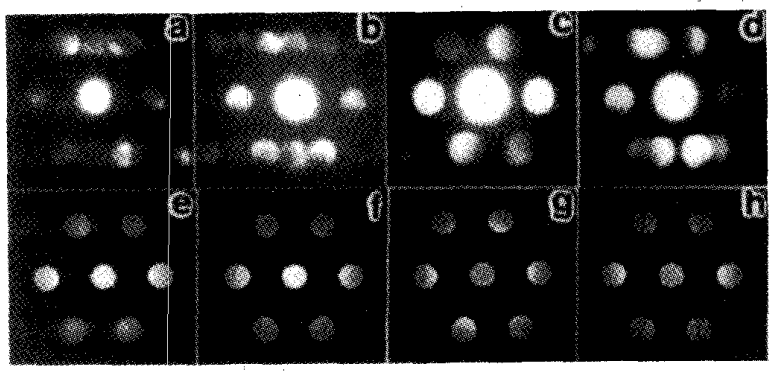

Figure 4. Comparison of TEM ( $a, b, c, d)$ and simulated (e , f, g,

h) $[11 \overline{2} 0]$ zone axis nanodiffraction patterns. (a) and (e) : random stacking, simulation was performed using a 9-layer stacking model, (b) and (f) Two-layer stacking AB, (c) and (g) : three layer stacking $\mathrm{ABC}$, (d) and (h) four layer stacking $\mathrm{ABAC}$.

When a close-packed atomic direction is aligned parallel to the electron beam, that is, when viewing in the [1120] direction, the relative stacking positions of $\mathrm{A}$ layer, $\mathrm{B}$ layer and $\mathrm{C}$ layer are projected at three levels with equal spacing. The HREM image, if taken near the Scherzer defocus (-43 nm for JEOL 2010) or the second pass band (-66 nm for JEOL 2010) is then a projection of the atomic columns. An attempt was made to take the HREM images on $[11 \overline{2} 0]$ zone axis (see the crystallite in Figure 1 marked c). To remove the noise, the high resolution micrographs were printed using an optical image processing system. A mask ( the Fourier filter) was put at the back focal plane of the image lens to allow the desirable beams to contribute to the image. One example of the optical diffraction pattern and filtered pattern is shown in Figure 5. As the diffraction spots split in one direction for different stacking modes, the mask shown in Figure 5 allows different stacking modes to be imaged with reduced noise. Two examples of the filtered HREM images are shown in Figure 6. The defocus setting of the HREM images was found to be about $-60 \mathrm{~nm}$. Each white dot represents an atomic column. The local stacking mode of three repeating unit cells of $\mathrm{ABAC}$ is found in Figure 6 (a) and 
three repeating unit cells of stacking $\mathrm{ABC}$ and four repeating unit cells of stacking $\mathrm{AB}$ are observed in Figure 6 (b). Random stacking modes are observed in both Figure 6 (a) and (b).

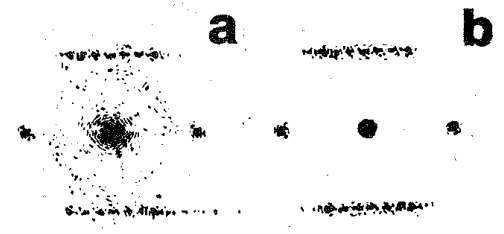

Figure 5. Comparison of optical diffraction patterns. (a) astaken from TEM negative, (b) filtered with the mask.

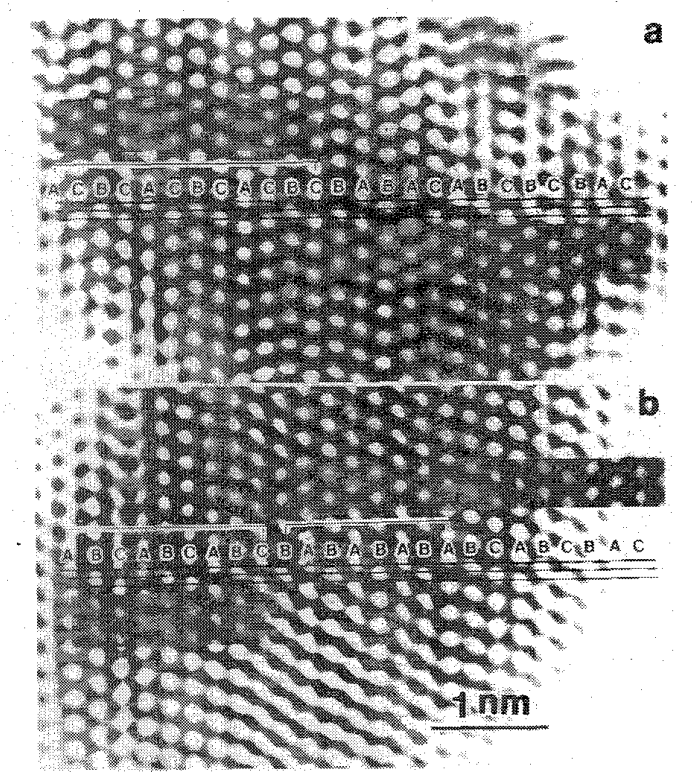

Figure 6. Two examples of [112̄0] zone axis HREM images taken from the crystallites in $\mathrm{CoSm}$ films. (a) three repeating unit cells of the stacking $\mathrm{ABAC}$ are observed. (b) three repeating unit cells of the stacking $A B C$ and four repeating unit cells of the stacking $A B$ are observed.

\section{DISCUSSION}

Since the crystallites contain some Sm, one may ask how the $\mathrm{Sm}$ atoms are distributed in the Co lattice. This question can be answered by examining the [0001] zone axis diffraction pattern where superlattice spots will appear if ordering occurs. Examples of superlattice spots in ordered structure of the $\mathrm{Cu}-\mathrm{Al}$ system can be found in Reference. 7. As no superlattice spots have been found in the [0001] zone axis pattern of the CoSm films, the Sm atoms are randomly distributed in the Co lattice.

One comment on the stacking mode of the crystallites is worth mentioning. Three nanodiffraction patterns in Figure 4 have been indexed using two layer stacking, three layer stacking, and four layer stacking models. However, this does not necessarily mean that the whole crystallite has the same mode of stacking, but only indicates that the stacking mode under the two nanometer probe can be specified by the suggested models. In fact, when a five nanometer probe was used, no clear patterns such as those in Figure 4 (b), (c) and (d) have been found. Therefore, when a whole crystallite is viewed, random stacking with local special stacking modes being present seems to be the proper description, which is exactly what is observed by HREM.

Close-packed structures with a random stacking sequence have been found in several alloy systems. In early 1960s,

researchers have found striations in the [1120] diffraction patterns of martensites resulted from quenching of some $\mathrm{Cu}-\mathrm{Al}$ alloys [7]. Recently, 9-layer stacking, 11-Iayer stacking and random stacking of close-packed structures were found in the laser cladding of $\mathrm{Ni}-\mathrm{Al}$ bronze on $\mathrm{Al}$ alloy $\mathrm{AA} 333$ [6,8,]. Therefore, close-packed structures with random stacking are meta-stable phases of quenched materials. The magnetic properties of the as-deposited CoSm films must be considered based on the deduced structural model.

\section{CONCLUSIONS}

The crystallites in the as-deposited CoSm films have a close-packed structure. Each crystallite has a particular stacking mode which consists of local random stacking, with occasional unit cells of two layer stacking $A B$, three layer stacking $A B C$, and four layer stacking ABAC.

* Research supported by ARPA/NSIC under Grant MDA 972-93$1-0009$.

\section{References}

1. E. M. T. Velu \& D. N. Lambeth, High density recording on SmCo/Cr thin film media, IEEE Trans. Magn., Mag-28, pp. 3249-3254, 1992.

2. D.J. Sellmyer, Z.S.Shan, Y. Liu, S.H.Liou, S. Malhotra and B.W. RobertsonMagnetic and structural properties of high coercivity nanocrystalline CoSm films with in-plane anisotropy, Acta Metallurgica, in press.

3. Y. Liu, B. Robertson, Z. S. Shan, S. Malhotra, M. J. Yu, S. K. Renukunta, S. H. Liou, and D. I Sellmyer, Nanostructures of $\mathrm{Sm}-\mathrm{Co}$ on $\mathrm{Cr}$ thin films, IEEE Transactions on Magnetics, Vol. 6. pp. 4035-4037, 1994.

4. Y. Liu, B.W.Roertson, Z.S.Shan, S.H.Liou and D.J. Sellmyer, Microstructure of the $\mathrm{Cr}$ underlayer and its effect on Sm-Co film, J. of Appl. Phys., April, 1995, in press.

5. P. A. Stadelmann, EMS- a software package for electron difraction and analysis and HREM image simulation in materials science, Ultramicroscopy, 21 , pp. 131-146,1987.

6. Y. Liu, J. Mazumder and K. Shibata, TEM study of martensite in laser clad Ni-Al bronze on Al alloy AA333, Metall. and Mater. Trans. A, 25A, pp. 37-46, 1994.

7. H. Sato, R. S. Toth and G. Honjo, Remarks on the structure of martensites in Cu-Al Alloys, Acta Met., 15, pp.1381-1396, 1967.

8. Y. Liu, J. Mazumder and K. Shibata, TEM WB and HREM crystal and defect study of martensite in laser clad Ni-Al bronze, Acta Metall. 42, pp. 1755-1762, 1994. 